\title{
VERZEICHNIS DER VON HERRN E. C. ABENDANON IN CELEBES GESAMMELTEN SÜSSWASSER-MOLLUSKEN
}

VON

J. H. KRUIMEL,

Assistent am Zoologischen Museum der Universität Amsterdam.

Mit 1 Tafel, einer Karte und 20 Textfiguren. 


\title{
Verzeichnis der von Herrn E. C. Abendanon in Celebes gesammelten Süsswasser-Mollusken
}

\author{
VON \\ J. H. KRUIMEL. \\ Assistent am Zoologischen Museum der Universität Amsterdam.
}

Mit 1 Tafel, einer Karte und 20 'Textfiguren.

EINLEITUNG.

Die von Herrn E. C. Abendanon auf seiner Reise in den Jahren 1909 und 1910 gesammelten Süsswasser-Mollusken liefern einen interessanten Beitrag zur Kenntnis der Seen von Central-Celebes.

In vorliegender Arbeit sind ausser diesen auch die Süsswasser-Mollusken aufgenommen, welche Herrn Abendanon später, im Jahre 1912, nachgeschickt wurden. Da aut der der Geologie gewidmeten Reise des Herrn Abendanon die Erforschung der Molluskenfauna nur Nebenzweck war, enthält die Ausbeute nur ein Teil der von Drs. P. und F. Sarasin in ihrem wertvollen Werke "Die Süsswasser-Mollusken von Celebes, 1898" beschriebenen Arten. Dennoch wurden einige netue Formen aufgefunden, indem einerseits der Towuti-See neue Arten lieferte, andererseits drei kleinere Seen (der Masapi-See, der Mahalona-See, der Wawontoa-See), die Herr Abendanon entdeckte, auf die Mollusken-Fauna hin durchforscht werden konnten. Diese drei kleinen Seen sind in der Nahe des Towuti-Sees gelegen. Nur der Mahalona-See hat durch den Tominanga-Fluss eine Verbindung mit dem Towuti-See. Dieser See ist damit zwischen den Matano-See und den Towuti-See eingeschaltet.

Die folgende Karte gibt einen Überblick über ihre Situation in Süd-Ost-Celebes.

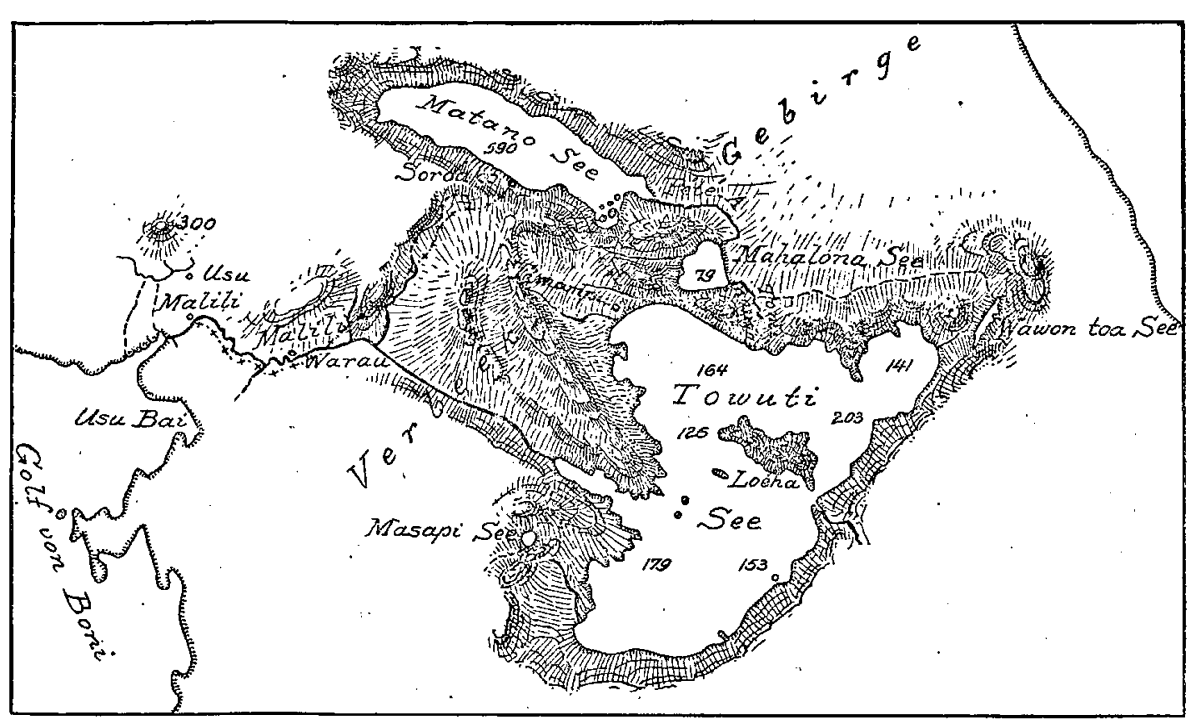

Karte der Süsswasser-Seen von Süd-Ost-Celebes. 
Das vorliegende Verzeichnis gibt also eine Ergänzung zu der bis heute bekannten Molluskenfauna von Celebes, zugleich enthält es einige Bemerkungen über den Wert der von Dr. P. und F. Sarasin vorgeschlagenen Einteilung von Miratesta celebensis Sar. in drei Varietäten.

Herr Dr. F. Sarasin war so freundlich mir einige Typen der von ihm beschriebenen Melanien auf meine Bitte hin zur Ansicht zu senden. Herrn M. M. Schepman verdanke ich manchen guten Rat. Für ihre freundliche Unterstützung sage ich beiden Herren herzlichen Dank.

\section{SYSTEMATISCHER TEIL.}

\section{PROSObRANCHIA.}

\section{A. Melaniidae.}

1. Melania toradjarum Sar.

Melania toradjarum P. und F. Sarasin, die Süsswasser-Mollusken von Celebes 1898 p. 14.

1 Exemplar. Das Gehäuse ist von den von P. und F. Sarasin beschriebenen Exemplaren verschieden indem seine Farbe nicht schwarz sondern braun ist. Diese Farbe ist wohl durch Abreibung entstanden.

Fundort: Posso-See, Central-Celebes.

\section{Melania palicolarum Sar.}

Melania palicolarum P. und F. Sarasin, die Süssw.-Moll. v. Celebes pg. 16.

103 Exemplare.

Fundort: Mahalona-See, Süd-Ost-Celebes.

y1 Exemplare. In Alkohol aufbewahrt.

Fundort: Towuti-See, Süd-Ost-Celebes.

27 Exemplare. In Alkohol aufbewahrt.

Fundort: Insel Loéha im Towuti-See, Süd-Ost-Celebes.

27 Exemplare.

Fundort: Insel Loéha im Towuti-See, Süd-Ost-Celebes.

1 Exemplar.

Fundort: Wawontoa-See, Süd-Ost-Celebes.

3. Melania masapensis n. sp. (Taf. IV, Fig. 13).

76 Exemplare. In Alkohol aufbewahrt.

Das Gehäuse ist schlank getürmt, gross, dunkel schwarz und dekolliert. Es ist also in Farbe und Form Melania palicolarum Sar. sehr ähnlich. Vorhanden sind 5-6 Umgänge, welche gebogen und durch deutliche Nähte von einander getrennt erscheinen. Ein Embryo zeigt die typischen Querrippen auf drei Umgängen, weiterhin sind die Umgänge, obgleich man eine Zeichnung von Quer- und Längsrippen ganz deutlich wie ein Muster wahrnehmen kann, glatt, wie beim erwachsenen Individuum, und treten nur die Anwachsstreifen hervor. Die Basis der letzten Windung zeigt etwa 9 kaum wahrnehmbare Spiralrippen (Längsrippen). Nabel geschlossen.

Die Mündung ist länglich oval. Ihre Spitze oben ebenso wie ihre halbkanalförmige Bucht unten sind nur sehr undeutlich ausgebildet. Sie ist also runder als bei Melania palicolarum. Vorgezogene, scharfe Aussenlippe und ansgerundeter Basalrand. Die Mündung ist innen von einem weisslich-blauen Ton überdeckt.

Der Deckel hat 10 Windungen, die letzte schnell verbreitert. 
Radula: Mittelzahn dem Mittelzahn von Melaniá palicolärum sehr åhnlich. Die Maasse der drei grössten Exemplare sind folgende:

$\begin{array}{cccc}\text { Schalenlänge. } & \text { Schalenbreite. } & \text { Mündungslänge. } & \text { Mündungsbreite. } \\ 48 \mathrm{~mm} . & 19 \mathrm{~mm} . & 17 \mathrm{~mm} . & 13 \mathrm{~mm} . \\ 45 \% & 17 \% / 2 \% & 16 \% & 11^{1 / 2} \\ 42 & 17 & 15 \% & 12\end{array}$

Fundort: Masapi-See, Süd-Ost-Celebes.

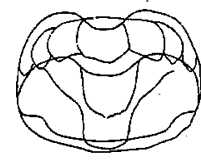

Mittelzahn der Radúla

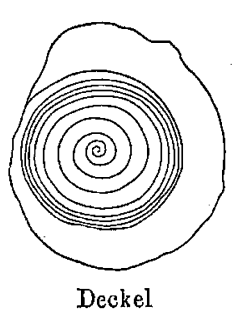

Deckel

von Melania masapensis n. sp.

4. Melania patriarchalis Sar.

Melania patriarchalis P. und F. Sarasin, die Süssw.-Moll. v. Celebes pg. 17.

81 Exemplare: 2 mit Winkel $5^{1}{ }_{2}, 4$ mit Winkel 5, 16. mit Winkel $4^{1} / 2,42$ mit Winkel 4, 10 mit Winkel $31 / 2$, 1 mit Winkel 3 , und 6 mit unbekanntem Winkel ${ }^{1}$ ).

Fundort: Matano-See, Süd-Ost-Celebes.

14 Exemplare: 1 mit Winkel 41/2, 11 mit Winkel 4, 2 mit Winkel $31 \frac{1}{2}$. Fundort: Westküste des Matano-See, Süd-Ost-Celebes.

24 Exemplare: 4 mit Winkel 5, 10 mit Winkel $4 \%_{2}, 9$ mit Winkel 4, 1 mit Winkel $31 / 2$. In Alkohol aufbewahrt.

Fundort: Soroako am Matano-See, Süd-Ost-Celebes.

142 Exemplare: 1 mit Winkel 5, 7 mit Winkel $4 \frac{1}{1} / 2,82$ mit Winkel 4 bis $4^{1}{ }^{1}, 46$ mit Winkel $3 \frac{1}{2}$ bis 4, 6 mit Winkel 3. In Alkohol aufbewahrt.

Fundort: Matano-See, Süd-Ost-Celebes.

\section{Melania gemmifera Sar.}

Melania gemmifera P. und F. Sarasin, die Süssw.-Moll. v. Celebes p. 20.

10 Exemplare: 4 mit Winkel 4, 4 mit Winkel $31 / 2,1$ mit Winkel 3, 1 mit Winkel $2^{1}{ }_{2}{ }^{1}$ ). In Alkohol aufbewahrt.

Radula. Die Radula ist noch nicht von P. und F. Sarasin beschrieben worden. Mittelzahn etwa $1^{1 / 2}$ mal breiter als lang. Die zwei oberen Ecken sind fast verschwunden, indem die ganze obere Hälfte des Zahns einen Bogen darstellt. Die beiden unteren Ecken springen um so deutlicher hervor, da ihre Spitze eingedrückt ist. Diese Zahnform ist also zweifelsohne vom Melania patriarchalis-Zahn verschieden. Auch schien es mir, dass die Zäckelchen kleiner sind als bei Melania patriarchalis, während die Hauptzacken um so deutlicher hervortreten. Eine mehr oder weniger nach aussen gerichtete Verlagerung kann jedoch die Grösse des Bildes der Zacken beeinträchtigen. Daher habe ich die Radula von drei Exemplaren untersucht. Sie ähneln einander ganz und gar.

Die Maasse der drei grössten Exemplare sind folgende:

$\begin{array}{cccc}\text { Schalenlänge. } & \text { Schalenbreite. } & \text { Mündungslänge. } & \text { Mündungsbreite. } \\ 85 \mathrm{~mm} . & 26 \mathrm{~mm} . & 18 \mathrm{~mm} . & 14^{1} / 2 \mathrm{~mm} . \\ 78 \% & 27 \% & 20 \% & 13^{1} / 2 \% \\ 69 " & 22 \% & 18 \% & 13^{1} / 2 \%\end{array}$

1) Für die Bedeutung des Begriffes „Winkel": vergleiche den Allgemeinen Teil, S. 233. 
Ich erwähne diese Maasse, weil P. und F. Sarasin scheinbar nur jüngere Exemplare bekannt geworden sind.

Fundort: Soroako Matano-See, Süd-Ost-Celebes.
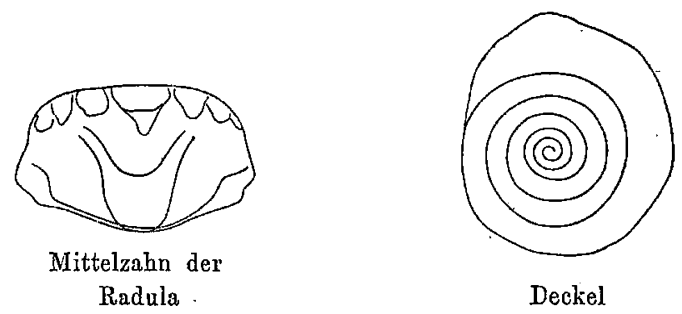

von Melania gemmifera Sar.

2 Exemplare 1 mit Winkel $31 / 2,1$ mit Winkel $3{ }^{1}$ ).

Fundort: Matano-See, Süd-0st-Celebes.

12 Exemplare: 2 mit Winkel 4, 5 mit Winkel 31/2, 2 mit Winkel 3, 3 mit Winkel $2^{1} / 2$.

Fundort: Westküste des Matano-Sees, Süd-Ost-Celebes.

3 Exemplare mit Winkel $4^{1} / 2$ sind eine Zwischenform zwischen Melania patriarchalis und Melania gemmifera. Sie sind wohl als Hybriden an zu sehen und beweisen nicht einen artlichen Zusammenhang zwischen beiden. Ihre grossen Unterschiede in Gehäuse, Deckel und Radula und die Seltenheit dieser Zwischenformen scheinen diese Behauptung zu beweisen.

Schale mit 18, 25 und 20 Querrippen (M: patriarchalis mit 30-40, M. gemmifera mit 11-14) auf der letzten. Windung. Winkel $4^{1} / 2$ (also M. patriarchalis ähnlich).

Mittelzahn der Radula nicht $1 \frac{1}{2}$ mal breiter als lang (also M. patriarchalis ähnlich). Die obere Zahnhälfte in der Mitte deutlich eingedrückt (also M. patriarchalis ähnlich) und nicht bogenförmig.

Deckel dem M. gemmifera-Deckel ähnlich.

Die unteren Ecken mit eingedrückter Spitze (also M. gemmifera ähnlich).

Fundort: Soroako Matano-See, Süd-Ost-Celebes.

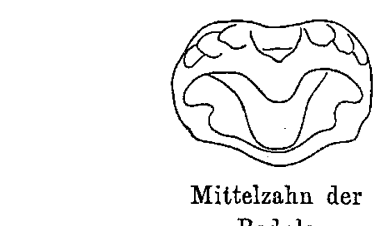

Radula

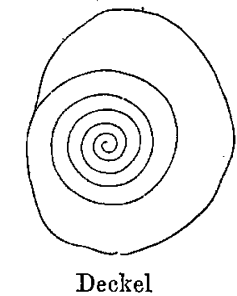

von der Zwischenform zwischen M. patriarchalis Sar. und M. gemmifera Sar.

6. Melania towutica n. sp. (Taf. IV, Fig. 8).

73 Exemplare: 60 mit Winkel 31/2, 13 mit Winkel 3. In Alkohol aufbewahrt.

Die Schale ist hoch getürmt, derb gebaut, dunkel oder gelblich braun, stark dekolliert und zerfressen. Sie ist der Schale von Melania patriarchatis Sar. ähnlich und ihr nahe verwandt. Die oberen Windungen sind mit einer weissen Sinterlage bedeckt. Zählbar sind 4-5 Umgänge. Die Querrippen sind kräftig doch in Körnchen zerfallen also nicht glatt. Sie sind mehr oder weniger geradelinig. Die Spirallinien der Basis nicht in Körnchen zerfallen. Nabel geschlossen.

Die Mündung zeigt die halbkanalförmige Bucht weniger deutlich als $M$. patriarchalis var. towutensis (P. und F. Sarasin die Süssw.-Moll. v. Celebes pg. 19).

Deckel mit 9 Windungen, die letzte schnell verbreitert.

Radula. Mittelzahn in der Konkavităt gemessen 11/2 mal breiter als lang, oben deutlich konkav. Die unteren Ecken mit etwas eingedrückter Spitze.

Die Maasse der drei grössten Exemplare sind folgende: 
221

\begin{tabular}{|c|c|c|c|}
\hline $\begin{array}{c}\text { Schalenlänge. } \\
44 \mathrm{~mm} . \\
42^{1 / 2} \% \\
43 \%\end{array}$ & $\begin{array}{c}\text { Schalenbreite. } \\
16^{1} / 2 \mathrm{~mm} . \\
16^{1} / 2, \\
16^{1} / 2\end{array}$ & $\begin{array}{c}\text { Mündungslänge. } \\
13 \mathrm{~mm} \text {. } \\
13 \text { " } \\
14^{1} / 2\end{array}$ & $\begin{array}{l}\text { Mündungsbreite. } \\
10^{2} / 2 \mathrm{~mm} \text {. } \\
14 \\
13{ }^{1} / 2 »\end{array}$ \\
\hline
\end{tabular}

Fundort: Insel Loéha, Towuti-See, Süd-Ost-Celebes.

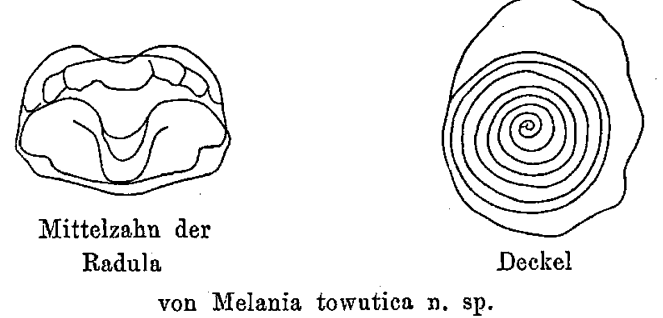

19 Exemplare: 1 mit Winkel 4⿺𠃊2, 6 mit Winkel 4, 5 mit Winkel $31 / 2,4$ mit Winkel 3. In Alkohol aufbewahrt.

Fundort: Towuti-See, Süd-Ost-Celebes.

\%. Melania patriarchatis var. towutensis Sar. (Taf. IV, fig. 6).

Melania patriarchalis var. towutensis P. und F. Sarasin die Süssw.-Moll. v. Celebes p. 19.

48 Exemplare: 6 mit Winkel 4\% 34 mit Winkel 4, 8 mit Winkel 31/2 ${ }^{1}$ ). In Alkohol auf bewahrt.

Diese Art ist bereits von P. und F. Sarasin beschrieben worden als eine Melania patriarchalis-Varietät. Meiner Ansicht nach hat sie, gleichwie Melania towutica, die vorhergehende Art, specifischen Wert. Doch schien es mir unnötig sie mit einem neuen Name zu belegen. Ich möchte jedoch zum Vergleich mit der vorhergehenden Art einige ihrer Eigentümlichkeiten betonen.

Zählbar sind 5-6 Umgänge. Die Querrippen sind kräftig und nicht in Körnchen zerlegt, also glatt. Sie sind niemals geradelinig, doch mehr oder weniger bogenförmig. Die Spirallinien der Basis nicht in Körnchen zerfallen.

Die Mündung mit deutlicher halbkanalförmiger Bucht.

Radula mehr oder weniger viereckig. Die unteren Ecken nicht eingedrückt.

Fundort: Insel Loéha, Towuti-See, Süd-Ost-Celebes.
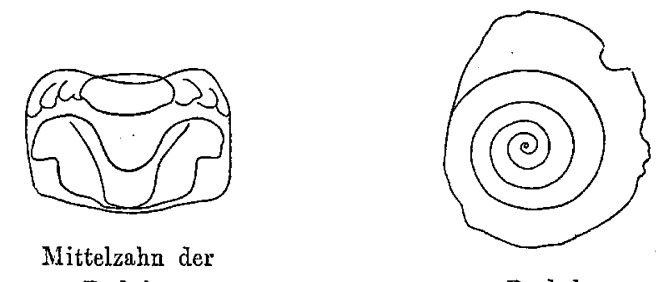

Mittelzahn der Radula Deckel

von M. patriarchalis var. towutensis Sar.

18 Exemplare: 5 mit Winkel $4 \frac{1}{2}, 8$ mit Winkel 4,3 mit Winkel $3_{12}^{1 / 2}, 2$ mit Winkel 3. In Alkohol aufbewahrt.

Fundort: Towuti-See, Süd-Ost-Celebes.

19 Exemplare: 2 mit Winkel $4^{1} / 2,9$ mit Winkel 4,8 mit Winkel $31 / 2$.

Fundort: Tominanga-Fluss beim Towuti-See, Süd-Ost-Celebes.

8. Melania mahalonica n. sp. (Taf. IV, Fig. 11).

10 Exemplare: 6 mit Winkel 4, 4 mit Winkel 31/8 ${ }^{1}$. In Alkohol aufbewahrt.

Die Schale ist hochgetürmt, sehr derb gebaut, dunkelbraun, dekolliert. Bis 8 Umgänge. Die Nähte zwischen allen Windungen tief eingeschnürt. Die Windungen sind stark gewölbt. Die Querrippen sind in Körnchen zerfallen also nicht glatt ausgebildet. Auch die Spiralrippen auf der Basis sind in Körnchen zerteilt. 
Die Mündung ist der von Melania patriarchalis ähnlich.

Deckel. Die Figur gibt das Bild eines jungen Deckels.

Radula. Mittelzahn, in der Konkavität gemessen, welche nämlich oben vorhanden ist, $11 / 2$ mal breiter als lang. Die unteren Ecken nicht eingedrückt. Der Mittelzahn dem von Melania patriarchalis ähnlich.

Die Maasse der drei grössten Exemplare sind folgende:

$\begin{array}{cccc}\text { Schalenlänge. } & \text { Schalenbreite. } & \text { Mündungslänge. } & \text { Mündungsbreite. } \\ 94 \mathrm{~mm} . & 29 \mathrm{~mm} . & 24 \mathrm{~mm} . & 15 \mathrm{~mm} . \\ 85 \% & 29 \% & 23^{1} / 2 & 15 \% \\ 70 \% & 25 \% & 19^{1} / 2 》 & 14 \%\end{array}$

Fundort: Mahalona-See, Süd-Ost-Celebes.

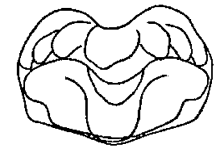

Mittelzahn der Radula

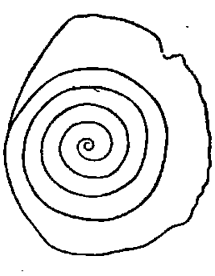

Deckel

von Melania mahalonica n. sp.

10 Exemplare: 4 mit Winkel $4 \frac{1}{2}, 4$ mit Winkel 4, 2 mit Winkel $3^{1} / 2$. Fundort: Mahalona-See, Süd-Ost-Celebes.

9. Melania mahalonensis n. sp. (Taf. IV, Fig. 12).

19 Exemplare: 15 mit Winkel 4, 3 mit Winkel $3^{1}{ }_{2}, 1$ mit Winkel $3^{1}$ ). In Alkohol aufbewahrt.

Die Skulptur der Schale ist der von Melania patriarchalis var. towutensis ähnlich, doch ist die Schale derb gebaut, hoch getürmt, dunkel-braun, dekolliert wie Melania patriarchalis. Bis 10 Umgänge sind vorhanden. Die Nähte zwischen allen Windungen mehr oder weniger eingeschnürt. Alle Windungen sind flach, oder nur die letzten kräftig gewölbt. Die Querrippen sind fast glatt, da die Spirallinien nur schwach ausgebildet sind. Die Spiralrippen auf der Basis der letzten Windung treten jedoch deutlich hervor, während an dieser Stelle die Querrippen verschwunden sind. Die Spiralrippen sind öfters in aneinander gereihte Körnchen zerfallen.

Die Mündung ist der von Melania patriarchalis ähnlich nur ist die halbkanalförmige Bucht mehr verlängert.

Deckel. Die Figur gịt das Bild eines jungen Deckels.

Radula. Mittelzahn ungefähr $1^{3 / 4}$ mal breiter als lang, oben deutlich konkav. Die beiden unteren Ecken mit etwas eingedrückter Spitze. Der ganze Zahn erscheint wie von links nach rechts ausgezogen.

Die Maasse der drei grössten Exemplare sind folgende:

$\begin{array}{cccc}\text { Schalenlänge. } & \text { Schalenbreite. } & \text { Mündungslänge. } & \text { Mündungsbreite. } \\ 89 \mathrm{~mm} . & 28 \mathrm{~mm} . & 24 \mathrm{~mm} . & 15 \mathrm{~mm} . \\ 87 \% & 28 \% & 23 \% & 15 \% \\ 83 \% & 281 / 2 \% & 24 \% & 15 \%\end{array}$

Fundort: Mahalona-See, Süd-Ost-Celebes.

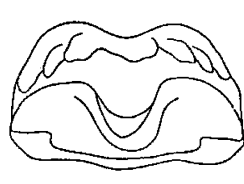

Mittelzahn der

Radula

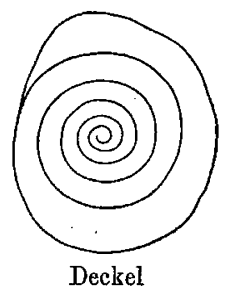

von Melania mahalonensis n. sp: 
28 Exemplare: 14 mit Winkel $4^{1} / s, 14$ mit Winkel 4.

Fundort: Mahalona-See, Süd-Ost-Celebes.

\section{Melania abendanoni n. sp. (Taf. IV, Fig. 9).}

36 Exemplare: 1 mit Winkel $4^{1} / 2,23$ mit Winkel 4, 12 mit Winkel $3^{1}{ }^{1}{ }^{1}$ ).

Das Gehäuse ist schlank getürmt, mittelgross, dünnschalig, dunkel braun, und dekolliert; vorhanden sind 5-6 Umgänge, welche ziemlich flach und durch untiefe Nähte getrennt erscheinen. Die Skulptur besteht aus Quer- und Spiralrippen, durch deren Kreuzung Körnchen entstehen. Die Querrippen sind jedoch deutlicher ausgebildet als die Spiralrippen. Die Zahl der Querrippen beträgt auf dem letzten Umgang 22-29. Die Basis der letzten Windung trägt 5-7 Spiralrippen (= Längsrippen), welche nicht von Querrippen in Körnchen zerteilt sind.

Die Mündung ist oval, basalwärts breit vorgezogen, ihre Basis ist nur sehr wenig halbkanalförmig ausgezogen. Die Farbe der Columella ist weisslich blau.

Deckel und Radula fehlten.

Die Maasse der grössten Exemplare sind folgende:

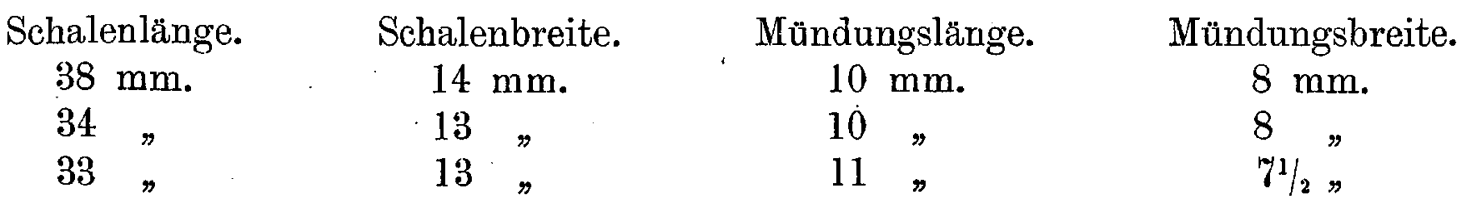

Fundort: Wawontoa-See, Süd-Ost-Celebes.

\section{Melania monacha Sar.}

Melania monacha P. und F. Sarasin, die Süssw.-Moll. v. Celebes pg. 21.

10 Exemplare.

In der Sammlung des Herrn Abendanon befinden sich nur mehr oder weniger beschädigte Schalen, sodass ich, obgleich ich über ein Exemplar der Herren Sarasin verfügen konnte, der Bestimmung dieser Schalen nicht ganz sicher bin. Deckel und Radula konnte ich nicht untersuchen. Die Querrippen stehen in unregelmässiger Entfernung und sind nicht alle vollständig von oben bis unten ausgebildet; daher sieht die Skulptur derb aus. Es mag sein, da P. und F. Sarasin über diese Art nur rom Matano-See berichten, dass meine Bestimmung unrichtig ist.

Fundort: Posso-See, Central-Celebes.

12. Melania perfecta Mousson.

\%4 Exemplare.

Es ist wohl sehr fraglich, ob diese Form Melania perfecta Mousson ist. Deckel und Radula fehlen. P. und F. Sarasin berichten über eine Melania perfecta Mousson im Kleide von tuberculata Müller aus dem in den Posso-See einmündenden Flusse Salokuwa (pg. 24). Die Schalen sind dieser Form sehr ähnlich.

Fundort: Insel Loéha im Towuti-See, Süd-Ost-Celebes.

\section{Melania sarasinorum n. sp. (Taf. IV, Fig. "y).}

14 Exemplare. In Alkohol aufbewahrt.

Das Gehäuse ist mittelgross, walzenförmig, dunkel violet-braun, dekolliert und zerfressen. Fast alle Windungen sind mit einer weissen Sinterlage bedeckt, nur die letzte Windung ist öfters unbedeckt. Zählbar sind höchstens noch 5 Umgänge, welche leicht gewölbt und durch ziemlich tiefe Nähte von einander getrennt sind. Die Querkostulierung fehlt vollständig; Anwachsstreifen sind vorhanden. Das ganze Gehäuse ist von deutlich hervorragenden Längsrippen umschlungen. Die letzte Windung zeigt deren etwa 18. Sie sind durch ungleich grosse Thäler getrennt. Es gibt deren dickere und dünnere. Nabel geschlossen.

Die Mündung ist ganz und gar der von Melania patriarchalis ähnlich: länglich oval, mit 
oberer Spitze. Aussenlippe scharf, unduliert, unten eine halbkanalförmige Bucht bildend. Diese Bucht ist beim grössten Exemplar weniger hervorragend als bei den kleineren. Der Schmelzbelag der Mündung ist weisslich-violett. Jüngere Individuen zeigen eine leicht angedeutete Nahtkante. Die Embryone haben auf den drei ersten Windungen die typische Querkostulierung.

Deckel mit 6 Windungen, die letzte schnell verbreitert.

Die Radula der von Melania patriarchalis ähnlich. Der Mittelzahn ist jedoch $12 / 3$ mal breiter als lang (bei Melania patriarchalis $1 \frac{1}{3}$ mal breiter als lang). Oben eine deutliche Konkavität.

Die Maasse der drei grössten Exemplare sind folgende:

\begin{tabular}{|c|c|c|c|}
\hline Schalenlänge. & Schalenbreite. & Mündungslänge. & Mündungsbreite \\
\hline $42 \mathrm{~mm}$. & $22 \mathrm{~mm}$. & $19 \mathrm{~mm}$. & $13 \% \mathrm{~mm}$. \\
\hline $36 \quad$ & $15^{1 / 2}$ & $13 \%$ & 9 \\
\hline $35 \%$ & $171 / 2 \%$ & $15 \%$ & 11 \\
\hline
\end{tabular}

Fundort: Insel Loéha im Towuti-See, Süd-Ost-Celebes.

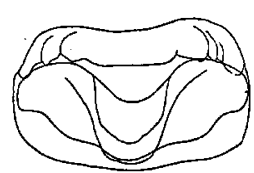

Mittelzahn der

Radula

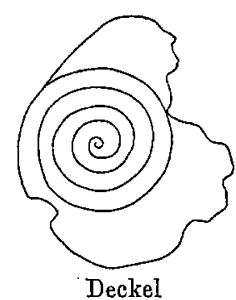

von Melania sarasinorum n. sp.

5 Exemplare. In Alkohol aufbewahrt.

Fundort: Towuti-See, Süd-Ost-Celebes.

12 Exemplare.

Fundort: Süd-Ost-Küste der grossen Insel Loéha im Towuti-See, Süd-Ost-Celebes.

14. Melania lalemae n. sp. (Taf. IV, Fig. 10).

44 Exemplare. In Alkohol aufbewahrt.

Das Gehäuse ist schlank getürmt, mittelgross, ziemlich dünnschalig, dunkel bräunlichschwarz, und dekolliert; vorhanden sind etwa 3-4 Ungänge, welche kaum gewölbt und durch deutliche Nähte von einander getrennt sind. Die Querkostulierung fehlt vollständig; nur mit der Loupe sieht man Querlinien. Anwachsstreifen sind vorhanden. Das ganze Gehäuse ist von Längslinien umschlungen. Die letzte Windung zeigt deren etwa 15. Erst an der Basis der letzten Windung treten durch Verbreiterung der Längslinien deutlich gebildete Längsrippen hervor. Hierdurch unterscheidet sich diese Art wesentlich von Melania sarasinorum. 'Es sind 6 bis 7 dieser Rippen vorhanden.

Die Mündung ist ganz und gar der von Melania patriarchalis ähnlich: länglich oval, mit oberer Spitze. Aussenlippe scharf unduliert, unten mit halbkanalförmiger Bucht. Der Schmelzbelag der Mündung ist weisslich-violett. Jüngere zeigen eine leicht angedeutete Nahtkante. Die Émbryone zeigen auf den ersten drei Windungen die typische Querkostulierung. Nabel geschlossen.

Deckel mit 5 oder 6 Windungen, die letzte schnell verbreitert.

Die Radula der von Melania patriarchalis ähnlich.

Die Maasse der drei grössten Exemplare sind folgende:

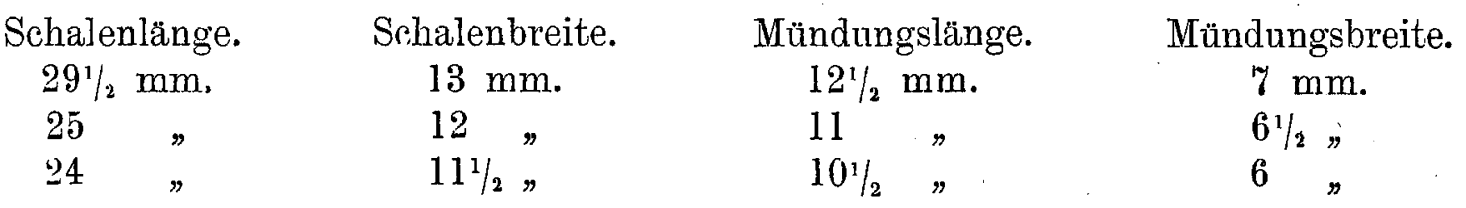

Fundort: Towuti-See, Süd-Ost-Celebes. 


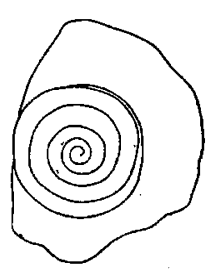

Deckel

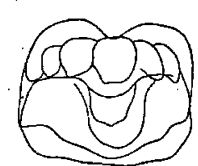

Mittelzahn der Radula

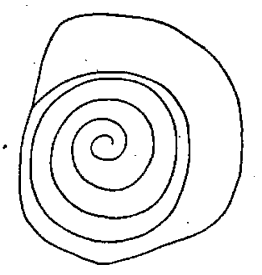

Deckel

von Melania lalemae n. sp.

15. Melania tominangensis n. sp. (Taf. IV, Fig, 14).

15 Exemp̄lare.

Das Gehäuse ist schlank getürmt, mittelgross, dünnschalig, dunkel schwarz und dekolliert; vorhanden sind 4-6 Umgänge, welche gebogen und durch deutliche Nähte getrennt erscheinen. Dex Embryo zeigt die typischen Querrippen auf drei Umgängen, weiterhin sind die Umgänge fast glatt, nur sehr schwach eine Teilung von Querlinien und Längsrippen vorzeigend. Die Basis der letzten Windung zeigt deutlich etwa 7 Spiralrippen (Längsrippen). Nabel geschlossen.

Die Mündung ist verlängert-eiförmig mit kaum merkbarer Andeutung der halbkanalförmigen Bucht, vorgezogener, scharfer Aussenlippe und ausgerandetem Basalrand. Innen ist die Mündung von einem blau-weisslichen Ton überdeckt.

Deckel und Radula fehlen.

Die Maasse der drei grössten Exemplare sind folgende:

\begin{tabular}{|c|c|c|c|}
\hline $\begin{array}{l}\text { Schalenlänge. } \\
35 \mathrm{~mm} \text {. }\end{array}$ & $\begin{array}{l}\text { Schalenbreite. } \\
10^{1} / 2 \mathrm{~mm} \text {. }\end{array}$ & $\begin{array}{l}\text { Mündungslänge. } \\
10 \mathrm{~mm} \text {. }\end{array}$ & $\begin{array}{c}\text { Mündungsbreite. } \\
\text { y mm. }\end{array}$ \\
\hline $32 »$ & 11 & 10 & 7 \\
\hline $31 \%$ & 11 & 11 & $y$ \\
\hline
\end{tabular}

Fundort: Tominanga-Fluss beim Towuti-See, Süd-Ost-Celebes.

16. Melania insulae sacrae Sar.

Melania insulae sacrae P. und F. Sarasin, die Süssw.-Moll. v. Celebes pg. 32.

9 Exemplare. In Alkohol aufbewahrt.

Fundort: Towuti-See, Süd-Ost-Celebes.

95 Exemplare.

Fundort: Süd-Ost-Küste der grossen Insel Loéha im Towuti-See, Süd-Ost-Celebes.

112 Exemplare.

Fundort: Nord-Küste der grossen Insel Loéha im Towuti-See, Süd-Ost-Celebes.

\section{1\%. Melania centaurus Sar.}

Melania centaurus P. und F. Sarasin, die Süssw.-Moll. v. Celebes p. 36.

14 Exemplare: 2 mit Winkel 4,6 mit Winkel 3, 4 mit Winkel 21/2, und 2 Winkel ${ }^{1}$ ) unbekannt.

Alle Exemplare sind mehr oder weniger beschädigt. Ein Individuum mit Schalenlänge 391/2 trägt 16 Querrippen auf der letzten Windung. Es sind hier 7 Umgänge vorhanden. Diese Zahl stimmt also gut mit der Angabe von P. und F. Sarasin (l. c. p. 3\%) überein. In Form und Schalendicke zeigt es aber mancherlei Anklänge an Melania gemmifera, in QuerrippenZahl ist es von ihr verschieden. Die Winkel-Variabilität passt zu Melania gemmifera. Es ist also schwer zu entscheiden, ob diese Exemplare zu Melania centaurus oder zu Melania gemmifera gehören. Es mag wohl sein, dass sie die beiden Species verbinden. Leider konnte ich Deckel und Radula nicht untersuchen. Jedenfalls wäre es aber wohl unerlaubt sie mit einem nenen Namen zu belegen. Der Fundstelle wegen habe ich sie bei Melania centaurus eingeteilt.

Fundort: Posso-See, Central-Celebes. 
B. Paludinidae.

1. Vivipara javanica v. d. Busch.

13 Exemplare.

Fundort: Posso-See, Central-Celebes.

3 Exemplare. In Alkohol aufbewahrt.

Fundort: Towuti-See, Süd-Ost-Celebes.

2. Vivipara persculptata Sar.

Vivipara persculptata P. und F. Sarasin, die Süssw.-Moll. v. Celebes pg. 62.

4 Exemplare.

Fundort: Posso-See, Central-Celebes.

C. Ampullariidae.

1. Ampullaria ampullacea Lam.

19 Exemplare. In Alkohol aufbewahrt.

Fundort: Towuti-See, Süd-Ost-Celebes.

6 Exemplare.

Fundort: Posso-See, Central-Celebes.

\section{I. Pulmonata.}

Limnaeidae.

1. Miratesta celebenses Sar.

Miratesta celebensis P. und F. Sarasin, die Süssw.-Moll. v. Celebes pg. 73-\%ั.

35 Exemplare.

Da P. und F. Sarasin bereits in ihrem wertvollen Werke die grosse Variabilität dieser Art betonen, bot sich eine gute Gelegenheit dar diese Variabilität mit der Ausbeute des Herrn Abendanon weiter zu untersuchen. P. und F. Sarasin haben, während sie diese Variabilität ähnlich wie solche z. B. von Limnaea auffassen, 3 Varietäten angenommen nämlich: robusta, ampullacea und gracilis. Am Ende der Beschreibung der dritten Varietät sagen sie: „Da wir sämtliche Varietäten in grösserer Zahl besitzen, so finden sich Stücke, welche einigermaassen den Uebergang von der einen zur anderen vermitteln, sodass wir uns nicht berechtigt glaubten, dieselben als eigene Arten zu beschreiben, so verschieden auch die extrem ausgebildeten Exemplare erscheinen". Mit meinem grösseren Material meine ich jetzt beweisen zu können, dass auch eine Einteilung in drei Varietäten nicht erlaubt ist, da die Maasse (Schalenlänge, Schalenbreite, Mündungslänge, Mündungsbreite) bei allen bekannten Individuen, und dies sind 47 Exemplaren (nämlich 34 messbaren von Abendanon und 13 von Sarasin), eine erhebliche Korrelation aufweisen und da bei dieser grösseren Zahl die Unterschiede in Farbe und Runzeln nicht mehr konstant auftreten.

Für meine Korrelations-Berechnungen habe ich die Formeln benutzt, wie sie JohannsuN ${ }^{2}$ ) angibt. Ich verweise also für ihre Bedeutung und ihren Wert auf dieses wertvolle Werk. Ich habe, wie gesagt, auf alle bekannte Exemplare Rücksicht genommen und verfügte bei meinen Berechnungen also über 47 Exemplare deren Maasse folgende sind:

2) Johannsen, Elemente der exakten Erblichkeitslehre 1909. 


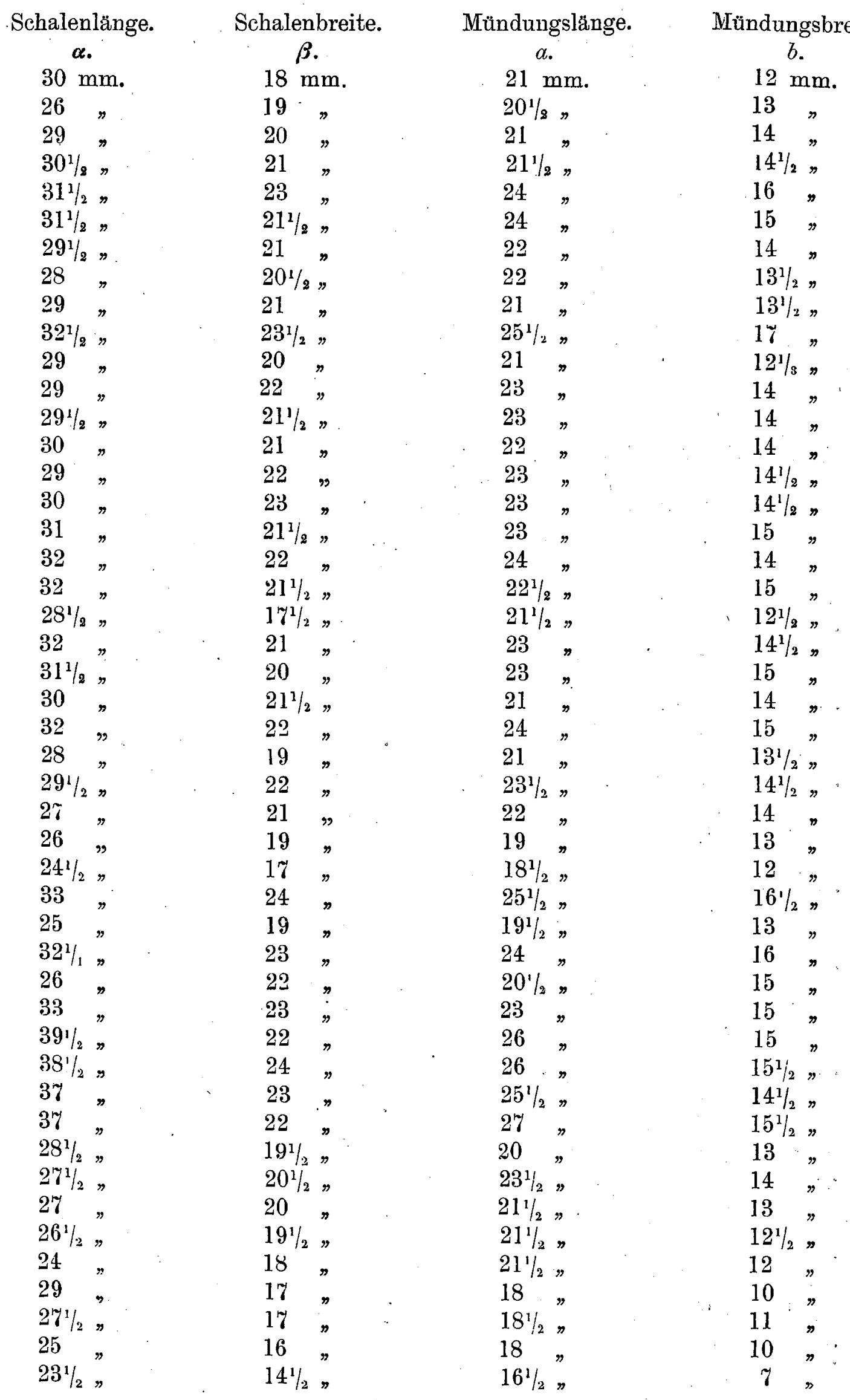

Die ersten 34 sind die Maasse der von Herrn Abendanon gesammelten Exemplare, die letzten 13 sind die Maasse durch P. und F. Sarasin (l. c. pg. 74 und. \%5) mitgeteilt. Die Schalenlänge wird hinfort mit $\alpha$ angedeutet. 
Die Schalenbreite wird, wie in unterstehender Figur, hinfort mit $\beta$ angedeutet.

Die Mündungslänge wird hinfort mit $a$ angedeutet.

Die Mündungsbreite wird hinfort mit $b$ angedeutet.

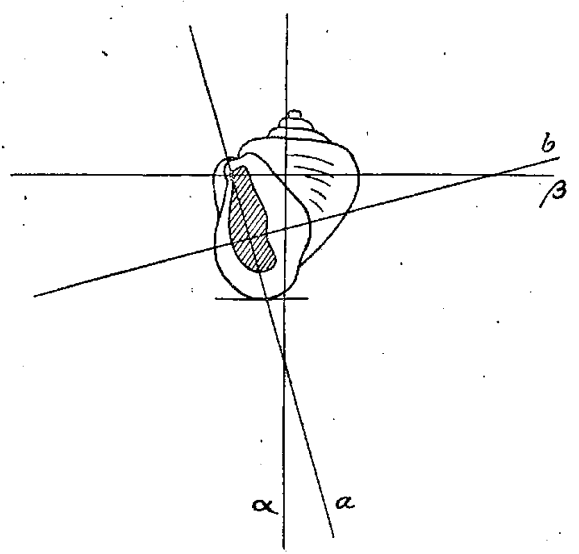

Die drei Varietäten Sarasins sind wohl durch ihre Beschreibung karakterisiert. Es müssen also die Tatsachen, das heisst die Maasse, mit dieser Beschreibung in Einklang sein, um ihre Einteilung zu rechtfertigen. Ich meine das Gegenteil beweisen zu können.

Aus der Beschreibung folgt ja für die drei Varietäten folgendes (Ich zitiere aus dem Buche pg. $\%$ u. f.):

Unter var. robusta:

$1^{\circ}$. ,Spira verhältnismässig kurz" also: $\frac{\alpha}{a}$ ist verhältnismässig klein.

$2^{\circ}$. "Mündung ist lang gezogen" also: $\frac{a}{b}$ ist verhältnismässig g ross.

$3^{\circ}$. Aus dem unter var. ampullacea ad $3^{\circ}$ Gesagten folgt: $\frac{\alpha}{\beta}$ ist verhältnismässig gross.

Unter var. ampullacea:

$1^{\circ}$. , kürzere Spira" also: $\frac{\alpha}{a}$ ist verhältnismässig seh $\mathrm{r}$ klein.

$2^{\circ}$. „Diese letztere (die Mündung) ist, der bauchigen Gestalt der Schale entsprechend, verhältnismässig breiter als die der vorigen Varietät (= var. robusta)":

also: $\frac{a}{b}$ ist verhältnismässig $\mathrm{klein}$.

3. , ,viel bauchigere Gestalt".

Unter var. gracilis:

also: $\frac{\alpha}{\beta}$ ist verhältnismässig $\mathrm{kl}$ ein.

$1^{\circ}$, , und mehr ausgezogener Spira",

also: $\frac{\alpha}{a}$ ist verhältnismässig gross.

$1^{\circ}$. „Die Mündung nimmt etwa zwei Dritteile der Gesammtschalenlänge ein (bei var. ampullacea mehr als $2 / 3$ )". also: $\frac{\alpha}{a}$ ist verhältnismässig gross.

$2^{\circ}$. Aus dem unter var. ampullacea ad $2^{\circ}$ Gesagten

folgt: $\frac{\alpha}{2}$ ist verhältnismässig gross.

$3^{\circ}$. „Von schlanker, viel minder bauchiger Gestalt als der vorige" (var. ampullacea). also: $\frac{\alpha}{\beta}$ ist verhältnismässig gross. 
Jeder Bruch ist ein Index, welcher die relative Gestalt einer Schale angibt, z. B.: $\frac{a}{b}=1$ bedeutet deshalb, dass die Mündungslänge $=a$ gleich gross ist wie die Mündungsbreite $=b$. Der Mündangsrand wird dann durch einen Kreis dargestellt. Die im Nenner stehende Zahl ist hier immer die grössere; doch sind die umgekehrten Brüche selbstverständlich auch als Indices verwendbar.

Die Indices der drei Varietäten sind also der Beschreibung nach von einander ganz und gar unabhängig und diese Unabhängigkeit, diese Selbstständigkeit der Varietäten, bedingt damit eine Selbstständigkeit der Maasse $(\alpha, \beta, a$ und $b)$ der $4 \%$ Individuen. Das heisst also: jede Varietät weist an und für sich eine Korrelation der Maasse auf; die 47 Individuen zusammen dürfen aber keine Korrelation der Maasse vorzeigen. Zur Kontrolle habe ich die Indices der 13 Exemplare von Sarasin berechnet und danach graphischen Vorstellungen angefertigt. Zum Beispiel folgen hier nur zwei Variationspolygone (Siehe S. 230) der Indices $a / \alpha$ und $\beta / \alpha$ der 13 Exemplare von P. und F. Sarasin.

Die Maasse und Indices dieser Exemplare sind folgende:

\begin{tabular}{|c|c|c|c|c|c|c|c|c|}
\hline & & $x \alpha$ & & $\alpha \beta$ & & $<a$ & $a / \alpha$ & $\beta / \alpha$ \\
\hline $\mathrm{N}^{\circ} 1$ & 79 & $\mathrm{~mm}$. & 44 & $\mathrm{~mm}$. & 52 & $\mathrm{~nm}$. & 0.66 & 0.56 \\
\hline 2. & 797 & , & 48 & " & 52 & , & 0.675 & 0.62 \\
\hline 3. & 74 & , & 46 & , & 51 & , & 0.69 & 0.62 \\
\hline 4. & 74 & ," & 54 & $"$ & 44 & , & 0.73 & 0.59 \\
\hline 5. & $57^{\circ}$ & , & 39 & ", & 40 & ", & 0.70 & 0.685 \\
\hline 6. & 55 & " & 41 & $"$ & 47 & " & 0.805 & 0.745 \\
\hline 7. & 54 & " & 40 & ", & 43 & , & 0.795 & 0.74 \\
\hline$\Rightarrow 8$. & 53 & , & 39 & " & 43 & , & 0.81 & 0.735 \\
\hline$\Rightarrow \quad 9$. & 48 & , & 36 & , & 43 & , & 0.895 & 0.75 \\
\hline " 10. & อ 8 & , & 34 & ", & 36 & , & 0.62 & 0.59 \\
\hline$" 11$. & 55 & $"$ & 34 & $"$ & $3 y$ & , & $0.6^{y}$ & 0.62 \\
\hline, 12. & 50 & " & 32 & $"$ & 36 & , & 0.72 & 0.64 \\
\hline " 13. & 47 & $"$ & 29 & $"$ & 33 & " & 0.70 & 0.62 \\
\hline
\end{tabular}

$\mathrm{N}^{\circ} 1-4$ ist var. robusta.

$\mathrm{N}^{\circ} 5$-9 ist var. ampullacea.

$\mathrm{N}^{\circ} 10-13$ ist var. gracilis.

Diese Variationspolygone (Siehe S. 230) weisen also eine unverkennbare Korrelation der Indices $\alpha / \alpha$ und $\beta / \alpha$ aller 13 Exemplare auf. Diese Korrelation aber ist, wie gesagt, der Annahme von P. und F. Sarasin, die drei Varietäten anerkennen, entgegengesetzt. Gleiches würden die Variationspolygone aller $4^{17}$ Exemplare vorzeigen. Eine exaktere Behandlung des Materials gibt jedoch die Korrelationsberechnung ${ }^{2}$ ). Ich habe daher die Berechnung der Korrelationskoeffizienten $(r)$ aller 47 Exemplare ausgeführt. Die Werte der Korrelationskoeffiziente sind folgende:

$$
\begin{aligned}
& \mathrm{l}^{\circ} \text {. für } \alpha \text { und } a r=0.840 \pm 0.043 \\
& 2^{\circ} . \quad, \alpha \quad \alpha, \beta \quad r=0.693 \pm 0.0 \% 6 \\
& 3^{\circ} . \quad, \beta \quad \beta \quad b \quad r=0.927 \pm 0.021 \\
& 4^{\circ} . \quad, a \quad, \quad b \quad r=0.848 \pm 0.041 \\
& 5^{\circ} . \quad " \quad \beta \quad, \quad a \quad r=0.558 \pm 0.039 \\
& 6^{\circ} . \quad, \alpha \quad, \quad b \quad r=0.646 \pm 0.085
\end{aligned}
$$

$r=1$ würde eine vollkommene Korrelation darstellen.

Es besteht also eine erhebliche Korrelation der Maasse. In $1^{\circ}, 2^{\circ}$ und $6^{\circ}$, wo $r$ am kleinsten ist, ist die Korrelation offenbar durch eine gleichartige Eigentümlichkeit beeinflusst worden. Das will sagen, dass $\boldsymbol{\alpha}$, die Schalenlänge, sehr wahrscheinlich eine Eigentümlichkeit besitzt, wodurch in den Făllen 1, 2 und 6, die im übrigen in der Grösse ihrer Korrelation von den übrigen Fällen nicht abweichen, diese Korrelation verkleinert wird. Diese Eigentümlichkeit tritt selbstständig auf ohne geradlinige Korrelation. Diese Eigentümlichkeit ist wohl das mehr oder weniger „Ausgezogen-sein" der Spira, m.a. W.: der Winkel, unter welchem die 
Basis (der Fuss) der wachsenden Schnecke sich in einer Spirale vom Gipfel entfernt, ist nicht gleich gross bei allen. Exemplaren, sondern weist an und für sich eine gewisse Variabilität auf. Ist der Winkel klein, dann nähert sich die Spirale einer flachen Spirale (wie bei Planorbis-Arten). ist er dagegen gross, dann nähert sie sich einer geraden Linie. Es ist zuvor leicht einzusehen, dass dieser Winkel mit der Mündung keine einfache geradlinige Korrelation aufweisen wird. Die Schalenlänge ist von dieser Variabilität wahrscheinlich beeinflusst, sodass ihre Korrelationskoeffizienten, welche sie mit den anderen Maassen hat, kleiner werden. Offenbar ist die Winkelvariabilität zu klein um auch die Schalenbreite zu beeinträchtigen. Die Schalenbreite $(\beta)$ weist daher wohl grosse Korrelationskoeffizienten auf.

Diese Korrelation aber streitet mit den Definitionen von P. und F. Sarasin.

Eine Verteilung nach Farbe und Runzeln lässt sich bei den 35 Exemplaren des Herrn Abendanon nicht durchführen.

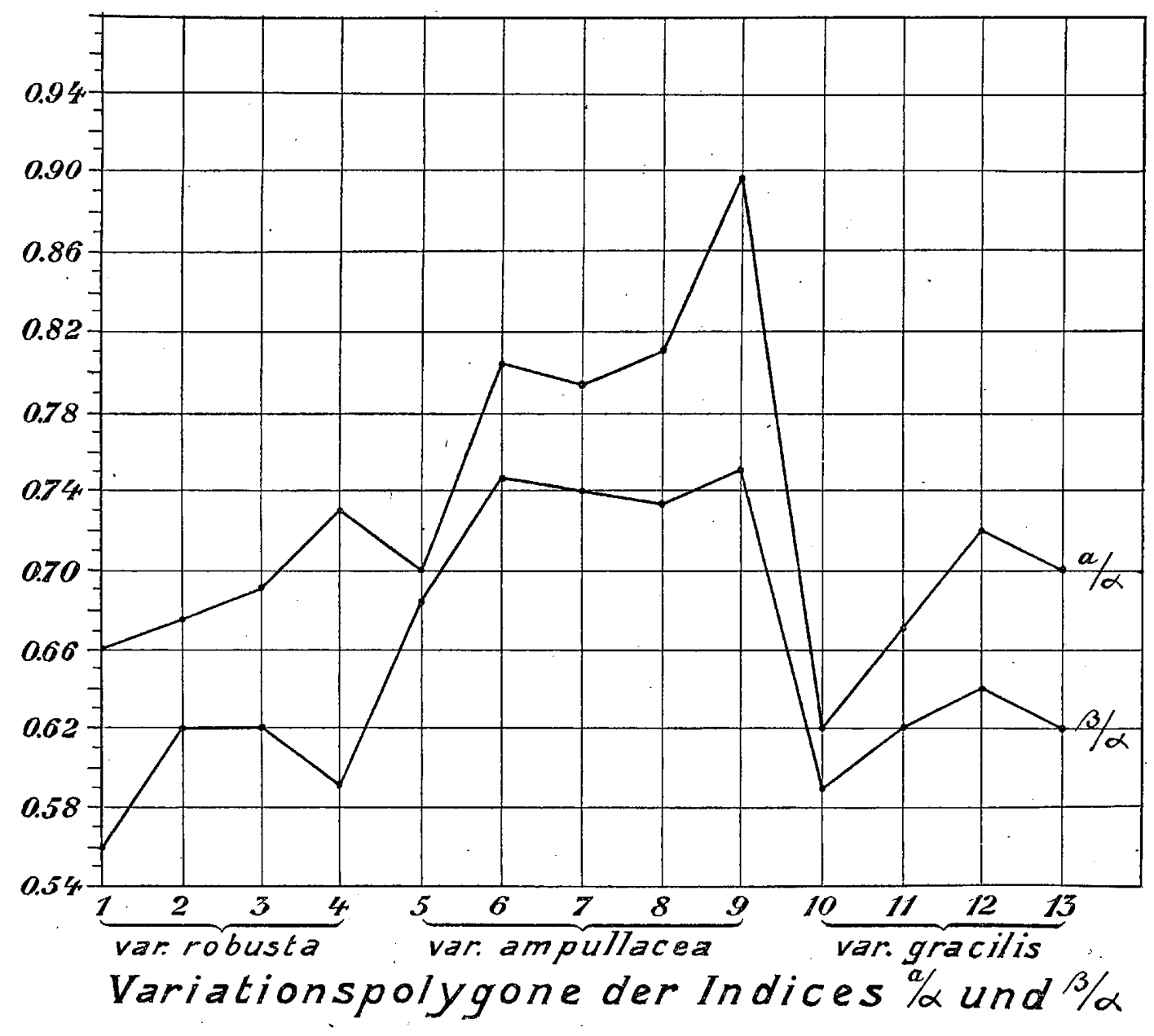

Vergleiche S. 229.

Hiermit glaube ich also bewiesen zu haben, dass man zur Annahme von drei Varietäten von Miratesta celebensis Sar. nicht berechtigt is. Es mag sein, dass diese Art eine Sammelart ist, doch ist bis heute das Material nicht zureichend, um eine unanfechtbare Einteilung auszuführen. Miratesta celebensis Sar. stellt sich überhaupt, z. B. in der Schalendicke, der Form der ohrartigen Falte u. s. w. als eine sehr variabele Art dar. Ein Versuch nach diesen Eigenschaften eine Einteilung $\mathrm{zu}$ machen habe ich nicht angestellt.

\section{Protancylus adhaerens Sar.}

Protancylus adhaerens P. und F. Sarasin, die Süssw.-Moll. v. Celebes p. 84.

1 Exemplar. In Alkohol aufbewahrt.

Fundort: Towuti-See, Süd-Ost-Celebes. 
3 Exemplare.

Fundort: Matano-See, Süd-Ost-Celebes.

3. Protancylus pileolus Sar.

Protancylus pileolus P. und F. Sarasin, die Süssw.-Moll. v. Celebes pg. 86.

2 Exemplare.

\section{LAMELLIBRANCHIATA.}

1. Corbicula matanensis Sar.

Corbicula matanensis P. und F. Sarasin, die Süssw.-Moll. v. Celebes pg. 92.

126 Exemplare. In Alkohol aufbewahrt.

Fundort: Matano-See, Süd-Ost-Celebès.

52 Exemplare.

Fundort: Matano-See, Süd-Ost-Celebes.

3 Exemplare.

Fundort: West-Küste Matano-See, Süd-Ost-Celebes.

48 Exemplare. In Alkohol aufbewahrt.

Fundort: Soroako, Matano-See, Süd-Ost-Celebes.

\section{Corbicula mahalonensis n. sp. (Taf. IV, Fig. 4).}

55 Exemplare. In Alkohol aufbewahrt.

Eine grosse an Corbicula matannensis Sar. sich anschliessende Form. Die Schale hat eine grobe Skulptur aus konzentrischen Rippen. Der Umriss der Schale ist hinten mehr gestreckt als bei Corbicula matannensis. Der Wirbel ist also in deutlich asymmetrischer Lage. Innen sind die Schalen fast ganz und gar von einem dunklen, violetten Ton überdeckt. Junge Individuen sind weniger gestreckt und der Form nach mehr Corbicula matannensis ähnlich, doch zeigen sie nicht die typische hellgelbe Farbe dieser Art. Sie sind bereits in ihrer Jugend olivenschwarzbraun.

Das grösste Exemplar ist $23,5 \mathrm{~mm}$. lang und 18,5 mm. hoch.

3. Corbicula possoensis Sar.

Corbicula possoensis P. unà F. Sarasin, die Süssw.-Moll. v. Celebes pg. 92.

19 Exemplare. In Alkohol aufbewahrt.

Fundort: Posso-See, Central-Celebes.

\section{Corbicula towutensis n. sp. (Taf. IV, Fig. 5).}

Eine mittelgrosse an Corbicula possoensis Sar. sich anschliessende Form. Der Umriss und die Skulptur der Schale sind ganz dieselben wie bei Corbicula possoensis. Alle Individuen zeigen aber (auch die jungen Stücke) einen hellvioletten Wirbel. Die Schale ist übrigens aussen hellgelb. Innen sind die Schalen mit prachtvollem violettem Ton überdeckt.

Die Maasse einiger Schalen sind folgende:

$\begin{array}{lc}\text { Schalenlänge. } & \text { Schalenhöhe. } \\ 16 \mathrm{~mm} . & 14 \mathrm{~mm} . \\ 15 " & 13 " \\ 141^{1} / 2 " & 13 " \\ 14 " & 12 "\end{array}$

Fundort: Towuti-See, süd-Ost-Celehes. 
5. Corbicula loéhensis n. sp. (Taf. IV, Fig. 2 und 3).

53 halbé Exemplare.

Eine mittelgrosse an Corbicula possoensis Sar. sich anschliessende, dünnschalige Form. Die Schale hat eine sehr feine Skulptur aus konzentrischen Rippen. Der Umriss der Schale ist hinten lang gestreckt, besonders bei älteren Exemplaren. Der Wirbel ist also sehr deutlich asymmetrisch gelegen. Die hellgelbe Farbe ist bei grösseren öfters von einem schwarzen Ton überdeckt. Innen sind die Schalen weisslich. Der Wirbel hat keinen violetten Ton.

Die Maasse einiger Schalen sind folgende:

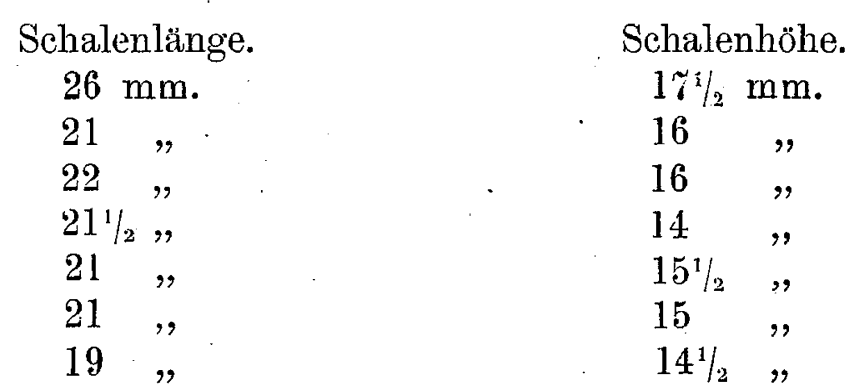

Fundort: Süd-Ost-Küste der grossen Insel Loéha im Towuti-See, Süd-Ost-Celebes.

6. Corbicule masapensis n. sp. (Taf. IV, Fig. 1).

185 Exemplare. In Alkohol aufbewahrt.

Eine mittelgrosse an Corbicula loéhensis (und somit an Corbicula possoensis Sar.) sich knapp anschliessende Form. Die Schale hat eine sehr feine Skulptur aus konzentrischen Rippen. Der Umriss der Schale ist hinten gestreckt, besonders bei jüngeren Exemplaren (Unterschied von Corbicula loéhensis). Der Wirbel ist bei erwachsenen asymmetrisch gelegen und fast immer zerfressen. Die Schale ist hellgelb mit weisslich-gelbem Wirbel. Innen sind die Schalen weisslich.

Die Maasse einiger Schalen sind folgende:

$\begin{array}{cc}\text { Schalenlänge. } & \text { Schalenhöhe. } \\ 27 \mathrm{~mm} . & 21 \mathrm{~mm} . \\ 25, " & 17 ", \\ 26 ", & 20 " \\ 20 " & 15^{1 / 2} " \\ 191 / 2 " & 14^{1 / 2} " \\ 18 ", & 14^{1 / 2} " \\ 18 " & 13^{1} "\end{array}$

Fundort: Masapi-See, Süd-Ost-Celebes.

\section{ALLGEMEINER TEIL.}

I. Allgemeine Bemerkungen über Melania patriarchalis und die an diese Species sich anschliessenden Melanien.

Melania patriarchalis Sar. und die ihr nahe verwandten Formen: Melania gemmifera, Melania towutica, Melania patriarchalis var. towutensis, Melania mahalonica, Melania mahalonensis, weisen eine ausserordentliche Menge von Modifikationen auf, welche vielleicht auf eben so viele Variationen, Subspecies oder Species hinweisen. Diese Veränderlichkeiten überdecken ein ander öfters, sie sind also transgressiv und es schien mir daher vergebliche Mühe ihrem Wesen nach zu forschen. Ich habe mich deshalb auf die Beschreibung der mehr typischen Formen beschränkt. Doch möchte ich auf eine Variabilität hinweisen, welche trans- 
gressiv ist, aber doch durch ihr Centrum (Mittel), dass bei jeder Form anders wo gelegen ist, eine auf anderen Eigentümlichkeiten sich gründende Einteilung bestätigen kann. Ich meine die im Systematischen 'Teil öfters angedeutete. WinkelvariabILITÄT.

Den Winkel einer Melania-Schale nenne ich den von zwei Flächen gebildeten Winkel, welche Flächen den Schalenseiten entlang gelegen sind und deren jede für sich senkrecht auf einer dritten, die Längsachse der Schale enthaltenden Fläche steht. Projiziert man diese beide Längsflächen auf die dritte, so bekommt man zwei Längslinien, welche einander unter demselben Winkel schneiden wie die beiden Flächen. Diesen Winkel habe ich bei jedem Exemplar mit der Ziffer: $2 \frac{1}{2}, 3,3 \frac{1}{2}, 4,4 \frac{1}{2}, 5$ oder $51 / 2$ angedeutet. Der Wert dieser Zahlen ist: $2^{1} / 2=14^{\circ}, 3=16^{1} /_{2}^{\circ} 3^{1}{ }_{2}=19^{\circ}, 4=23^{\circ}, 4^{1} / 2=26^{\circ}, 5=29^{\circ}, 5^{1} / 2=32^{\circ}$. Selbstverständlich sind diese Messungen nicht mit mathematischer Genauigkeit verrichtet worden. Ich erwähnte sie nur um im ganzen und grossen das Variationsbild des Winkels anzugeben und die Aufmerksamkeit auf ihr Dasein (die Winkelvariabität) zu lenken. Daher war es auch besser die Ziffern und nicht die genauen Gradzahl zu erwähnen, weil ja die Ziffern nur einen geschätzten Wert vorstellen und daher zum Beispiel $21 / 2$ ausser $14^{\circ}$ auch $131 /{ }^{\circ}$ oder $141_{2}^{\circ}$ bedeuten kann. Diese Abweichungen waren jedoch immer sehr klein. Die Winkelvariabilität ist umso merkwürdiger, als bei Exemplaren einer Fundstelle Schalen mit Winkel $5^{1} / 2$ und 3 gefunden worden sind. Der Schalenwinkel schwankt dann zwischen $32^{\circ}$ und $16^{1} 2_{2}^{\circ}$.

Beim Bestimmen der Winkelvariation, bemerkt man sofort, dass die Längslinien oder Längsflächen, welche man der Schale entlang sich denken kann, keine geraden Linien bezw. Flächen sind. Sie sind etwas, aber meistens nur sehr wenig, gebogen, sodass der Gipfel der 'Schale einen grösseren Winkel aufweist als die Basis derselben. Demgemäss haben die Embryonalschalen einen grösseren Winkel als die erwachsenen. Die ersten drei Windungen bei aus der Mutter befreiten Embryonen, sind in dieser Hinsicht wohl sehr typisch und selbstständig. Hier findet man öfters einen Winkel der $90^{\circ}$ noch überschreitet. Die folgende Windungen aber nehmen bald den fast konstanten Winkel an, welcher also immer kleiner ist. Bei sehr alten erwachsenen Exemplaren weisen die letzten Umgänge einen kleineren Winkel auf.

Die Winkeldifferenz scheint von einer Längendifferenz begleitet zu sein: je grösser der Winkel, desto kleiner die Schalenlänge und desto kleiner die Zahl der Windungen. Als Beispiel will ich hierfür die Angaben von zwei Exemplaren anführen:

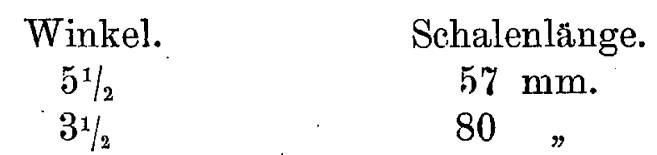

Windungen.
6
8

Bei beiden ist die Mündung gleich gross. Es mag sein, dass diese drei Faktoren durch eine gewisse Korrelation verkettet sind. Vielleicht ist die Grösse der Mündung kein richtiger Massstab, um das Alter eines Tieres zu bestimmen. Die Variabilität würde sich dann in anderer Weise zeigen. Doch ist es wohl am wahrscheinlichsten, dass die Grösse der Mündung, welche der Dicke des Tieres entspricht, eine richtige Schätzung seines Alters gewährt.

Andere Variabilitäten bei Melania patriarchalis und Verwandten betreffen die Querund Längsrippen. Diese sind am deutlichsten an der letzten Windung. Oft findet man zwischen den Spiralrippen der Basis intermediär viel schwächer ausgebildete Rippen, welche mit den derberen abwechseln, oft fehlen sie. Die Längsrippen der unteren Hälfte der ietzten Windung zerteilen sich nicht bei allen Exemplaren in Längsreihen von Körnern. Man findet eine grosse Variabilität in Skulptur, Breite und Zahl der Quer- und Längsrippen. Die Wölbung der Windungen ist sehr verschieden.

Es kann daher, bei einer so grossen Variabilität, wenig Verwunderung wecken, dass in den eben erst durch Herrn Abendanon erfoschten Seen sich nun auch sofort neue locale Varietäten vorfanden. Die Art dieser localen Rassen ist wohl, ihrer Radula und ihres Operculum wegen, am wahrscheinlichsten eine spezifische. Doch kann man das selbstverständlich an dem toten Material bei verhältnismässig kleinem Unterschiede nicht sicher beweisen. 
II. Allgemeine Bemerkungen über die Seen von Central-und Süd-Ost-Celebes und ihre Molluskenfauna.

Aus dem systematischen Teil und aus der Karte der Einleitung pg. 217 geht hervor, dass Herr Abendanon neben dem Posso-See, Matano-See und Towuti-See noch drei kleinere Seen erforscht hat, nåmlich den Mahalona-See, den Masapi-See und den Wawontoa-See.

Ich möchte zum Schluss einen Ueberblick über die Molluskenfanna aller dieser Seen geben.

$$
\text { 1. Posso-See. }
$$

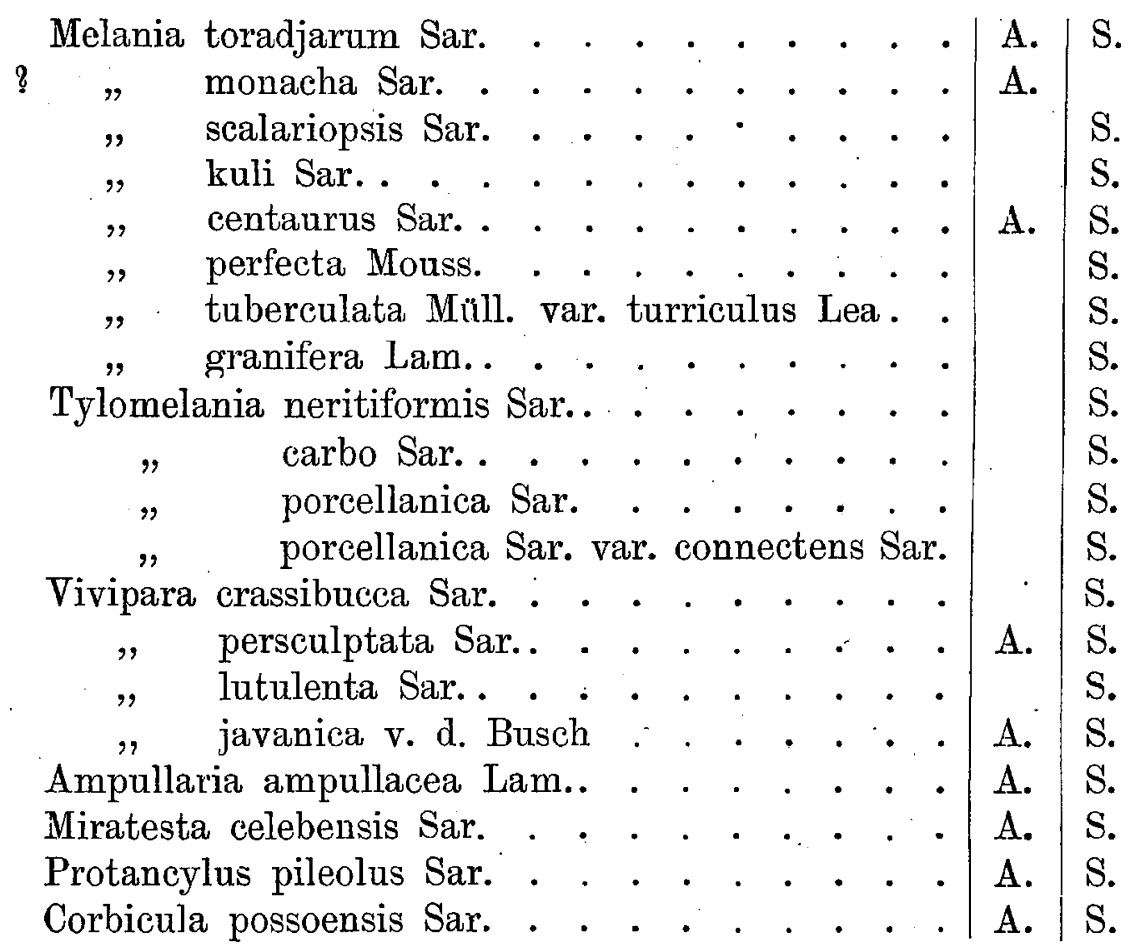

\section{Matano-See.}

\begin{tabular}{|c|c|c|c|c|c|c|}
\hline elania & halis Sar. . & & & & & \\
\hline , & gernmifera Sar. & & & & • & A. \\
\hline$"$ & monacha Sar. & & & & & \\
\hline ", & molesta Sar. & • & & & & \\
\hline & zeamais Sar. & & & & & \\
\hline Vivipas & rudipellis Sar. & ${ }^{\circ}$ & & & • & \\
\hline Protancy & dhaerens Sar. & & & & & A. \\
\hline orbicula & a matannensis Sar. & & & & . & A. \\
\hline
\end{tabular}

\section{Towuti-See.}

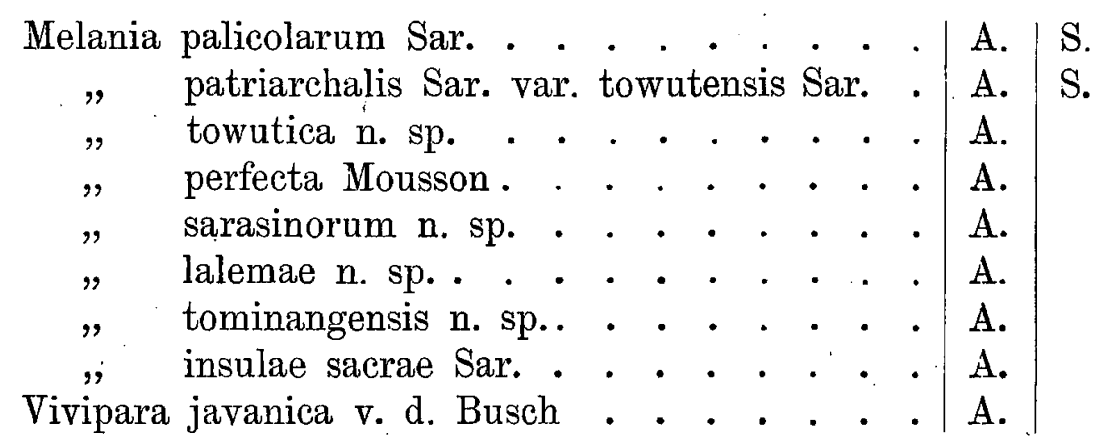




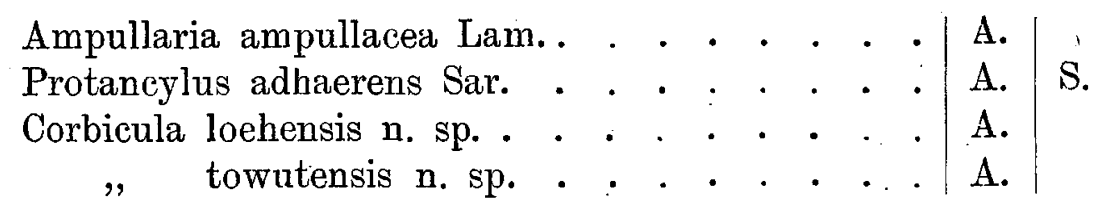

4. Mahalona-See.

Melania palicolarum Sar. . . . . . . . . A.

" mahalonica n. sp.. . . . . . . . A.

" mahalonensis n. sp. . . . . . . . A.

Corbicula mahalonensis n. sp. . . . . . . . A.

5. Masapi-See.

Melania masapensis n. sp. . . . . . . . . A.

Corbicula masapensis n. sp. . . . . . . . . .

6. Wawontoa-See.

Melania palicolarum Sar. . . . . . . . . A.

„ abendanoni n. sp. . . . . . . . . A.

A bedeutet: in der Ausbeute Abendanons, S: in der Ausbeute Sarasins.

Die Prophezeiung von P. und F. Sarasin: „Es ist ja wohl möglich, dass sich mit genauerer Kenntnis dieses Verhältnis (die Verschiedenheit der Molluskenfauna zwischen dem Posso- einerseits und dem Matano- und Towuti-See andererseits) etwas ändern wird, und dass sich einzelne gemeinsame Formen finden werden", hat sich also bestätigt. Ampullarıa ampullacea, Vivipara javanica und vielleicht Melania monacha und Melania perfecta kommen im Posso-See und im Matano- oder Towuti-See vor. Dennoch lässt sich eine Einteilung in zwei Gebiete, der Molluskenfauna nach, anfrecht erhalten. Die nahe Verwandtschaft der Corbiculae des Masapi- und Towuti-Sees mit der des Posso-Sees einerseits und der Corbiculae des Matano- und MahalonaSees andererseits ist schwer zu verstehen. Die grosse Verschiedenheit zwischen der Ausbeute von P. und F. Sarasin und der Ausbeute Abendanons beweist aber, das auch Abendanon den Formenreichtum wohl noch nicht erschöpft hat. Der Wawontoa-See ist durch Melania palicolarum mit dem Towuti-, Mahalona- und Matano-See verbunden. Die Daten sind leider nicht zureichend, um geographische Speculationen zu berechtigen. Es ist jedenfalls bemerkenswert, dass jeder See seine locale Varietäten von Melanien und Corbiculae hat. Die Seen sind daher wohl längere Zeit als Seen von einander getrennt gewesen, denn, obwohl sie durch einen Fluss verbunden sind, war diese Verbindung offenbar nicht genügend, um eine einheitliche Molluskenfauna hervorzurufen. Der Matano-, Mahalono- und Towuti-See beweisen diese Ansicht. 


\section{TAFELERKL ̈̈RUNG.}

TAFEL IV.

Fig. 1. Corbicula masapensis n. sp. S. 232.

Fig. 2, 3. Corbicula loéhensis n. sp. S. 232.

Fig. 4. Corbicula mahalonensis n.sp. S. 231.

Fig. 5. $\quad$ Corbicula towutensis n. sp. S. 231.

Fig. 6. Melania patriarchalis var. towutensis Sar. S. 221.

Fig. \%. Melania sarasinorum n. sp. S. 223.

Fig. 8. Melania towutica n. sp. S. 220.

Fig. 9. Melania abendanoni n. sp. S. 223.

Fig. 10. Melania lalemae n. sp. S. 224.

Fig. 11. Melania mahalonica n. sp. S. 221.

Fig. 12. Melania mahalonensis n.'sp. S. 222.

Fig. 13. Melania masapensis n. sp. S. 218.

Fig. 14. Melania tominangensis n. sp. S. 225 . 


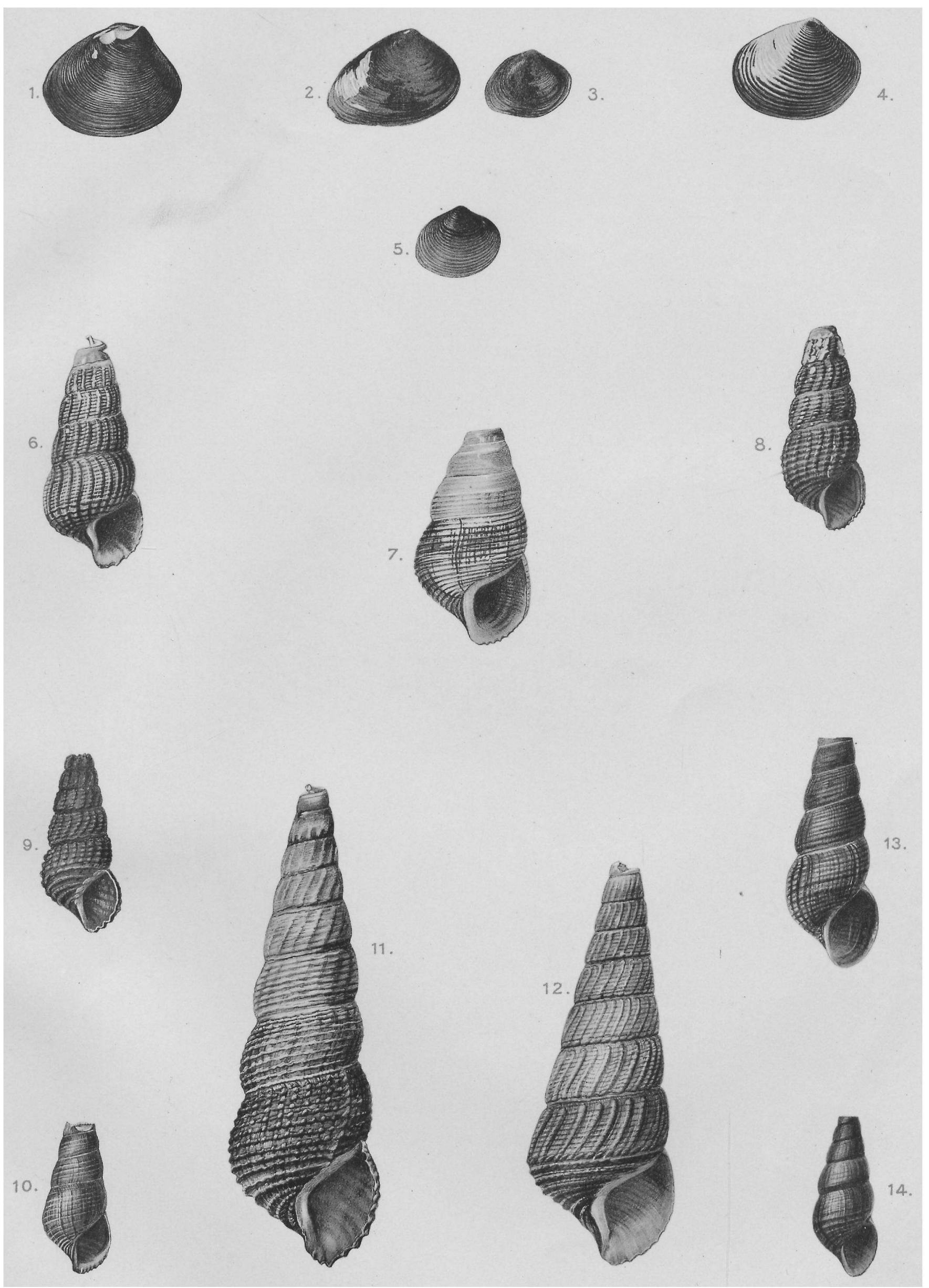

